\title{
PERFORMANCE OF UREA/NaOH AS A GREEN SOLVENT IN DISSOLVING RECYCLED CELLULOSIC FIBER FINES RESIDUES
}

\author{
SHAKIBA BAGHERI, MEHDI RAHMANINIA and RABI BEHROOZ \\ Wood and Paper Science and Technology Department, Faculty of Natural Resources, \\ Tarbiat Modares University, Noor, Iran \\ $\bowtie$ Corresponding author: M. Rahmaninia,rahmaninia@modares.ac.ir
}

Received August 10, 2021

\begin{abstract}
Using lignocellulosic materials for producing more value-added bioproducts is an attractive mission. Fiber fines, which represent an important part of the wastes generated by paper recycling mills, have been considered in the current research. Dissolving these lignocellulosic residues in environmentally friendly and inexpensive solvents can be a great achievement. For this purpose, the performance of urea/sodium hydroxide in dissolving printing and writing pulp (RPW) fines was investigated. Although using sodium hydroxide alone had a positive effect on the dissolution of recycled printing and writing pulp (RPWP) fines, the addition of urea increased the dissolution of fines from $23 \%$ to $56 \%$. Different levels of urea consumption had no significant effect on the dissolving process. The performance of the urea/sodium hydroxide system in dissolving fines suspensions with different concentration (1, 3 and 5\%) showed that reducing the concentration leads to an increase in fines dissolution (56,36 and 7\%, respectively). The results of FTIR confirmed the presence of cellulose without any hemicelluloses and lignin in the dissolving part. The results of X-ray diffraction analysis of soluble cellulose showed that the type-I cellulose structure probably changed to type-II cellulose. No reduction in the DP of dissolved cellulose and the integrated structure of the final cellulosic film confirmed by the FE-SEM images affirmed the successful dissolution of the RPWP fines in this system.
\end{abstract}

Keywords: dissolved cellulose, fiber fines, green solvent, paper recycling mill, urea/sodium hydroxide aqueous solution

\section{INTRODUCTION}

Nowadays, environmental concerns are widely taken into consideration in research and development in different areas. In this regard, biopolymers from renewable resources have attracted much attention. ${ }^{1}$ Cellulose, as the most abundant natural polymer on the planet, has been a topic of thorough research. ${ }^{2-3}$ Cellulose is a linear biopolymer consisting of $\beta-(1 \rightarrow 4)$ Dglucopyranose monomers. This biopolymer containing three hydroxyl groups $(\mathrm{OH})$ in each monomer can form strong intermolecular and intramolecular hydrogen bonds. ${ }^{4-5}$ Cellulose can be applied for making different products, such as different paper grades, cellulose derivatives (ether and ester) and regenerated materials (fibers, films, food packaging, membranes, sponges, hydrogels etc.). ${ }^{6}$ This valuable biopolymer can be obtained from various wood (softwoods, hardwoods) and non-wood resources (cotton linter, bamboo, straw etc.). ${ }^{7}$ Besides, wastepaper has attracted a lot of attention as an important part of solid wastes. Furthermore, the ever-increasing demands for cellulosic materials, the concerns about deforestation, developing environmental laws and policies, and the interest in the reduction of production costs have made wastepaper recycling more attractive. ${ }^{8-10}$

As most wastepaper goes through paper recycling mills for reproducing different recycled paper grades, the recycling process can damage lignocellulosic/cellulosic fibers, particularly, it reduces their length. ${ }^{4,11-12}$ This means that the fibers' fines content (tiny fiber particles, less than 76-micrometer length) will increase drastically, especially by increasing the number of recycling times. As these large amounts of fines may play an improper role in the papermaking process (damaging the structure of the paper by reducing the interlocking of the paper web, decreasing the drainage of water from the paper web, increasing the cost of production etc.), so most of paper recycling mills try to separate these fiber fines residues from the process and wastewater to improve the process and product quality. ${ }^{13}$ These separated fiber fines usually form a huge amount of residues in recycling mills, and their disposal becomes a major concern. Considering the abovementioned points, it seems that using this waste 
for producing value-added cellulosic products can be completely economical and also an environmental mission.

An important procedure for using cellulosic/lignocellulosic materials in value-added products is dissolving them. However, the main problem here is the limited cellulose/lignocellulose dissolution in common solvents, such as water, or organic ones. ${ }^{3}$ This is caused by the presence of strong intra- and intermolecular hydrogen bonds in cellulose. ${ }^{14}$ The traditional approach to regenerated cellulose products is mainly based on the viscose process, which is still common, but this process causes environmental pollution because of the use of $\mathrm{CS}_{2}$ and because of releasing harmful by-products into the environment. Therefore, finding an environmentally friendly cellulose solvent is an important issue in the production of regenerated cellulosic material and cellulose derivatives. ${ }^{15}$ Different solvent systems, such as $\mathrm{CS}_{2} / \mathrm{NaOH}$, LiCl/DMAc (lithium chloride/N, N-dimethyl acetamide), NMMO (N-methyl morpholine-Noxide)/water, ionic liquids, alkaline aqueous solutions and urea/sodium hydroxide aqueous solutions, have been developed for cellulose dissolution. ${ }^{2,4,17-18}$ Among these dissolving methods, the aqueous solution of urea/sodium hydroxide has several advantages, such as low costs, non-volatility, low toxicity, environmental friendliness etc. ${ }^{15}$

As previous studies have shown that the history of cellulosic fibers can affect their dissolution in different dissolving systems, ${ }^{19-20}$ the performance of urea/sodium hydroxide in dissolving recycled cellulosic fiber fines (as an important part of recycling mills' waste, which has passed through papermaking and paper recycling processes and its characteristics have experienced several changes, such as decreasing dimensions because of mechanical forces, increasing crystallinity of cellulosic fibers because of drying, hornification, etc.) was considered. In this respect, the current research investigates the performance of the urea/ $\mathrm{NaOH}$ aqueous system as a green solvent in dissolving recycled cellulosic fiber fines residues.

\section{EXPERIMENTAL Materials}

The RPWP fines were prepared from unprinted printing and writing waste papers. The required chemicals, including urea $(99.9 \%$ purity), sodium hydroxide (99.9\% purity) and acetone (99\% purity), were purchased from Merck Co. (Germany).

\section{Methods}

\section{Preparation of fines}

According to TAPPI standard, T $200 \mathrm{sp}-01$, RPW was prepared with $1.5 \%$ consistency, using a Valley beater. A Dynamic Drainage Jar (DDJ) equipped with a 200 mesh screen was used to separate the fines (particles passing through the 200 mesh screen (76micrometer length) according to TAPPI T261 cm-00 standard are called fines, which includes fiber fines and fillers) from the pulp suspension. As the fiber fines should be collected for continuing the dissolving process, the total fines were screened on a 400-mesh screen, which helped to remove fillers passing through it and the fiber fines remaining on the screen.

\section{Determination of chemical composition of fine fibers}

As the printing and writing paper, used in this research, was made of completely bleached pulp, there was no more lignin in it. For analysis of other existing components, firstly, the percentage of soluble materials in organic solvents was measured according to TAPPI standard T $204 \mathrm{~cm}-07$. Also, the ash content of the pulp was determined according to TAPPI standard $\mathrm{T}$ $211 \mathrm{om}-07$, which is a good indicator of the remaining filler in fiber fines after washing on the 400 mesh screen. The remaining part of fiber fines was considered as holocellulose (cellulose and hemicelluloses).

\section{Dissolution of fiber fines}

Considering the effect of sodium hydroxide for dissolving fiber fines, firstly the recycled fines were treated by aqueous sodium hydroxide solution with a ratio of 7:93 (sodium hydroxide:water).

Also, applying urea in aqueous alkaline for dissolving fiber fines was evaluated by using a sodium hydroxide/urea/water mixture with a ratio of $7: 4: 89$. For this purpose, before treating, the mentioned solving system was pre-cooled at below $-10{ }^{\circ} \mathrm{C}$ temperature and immediately mixed with RPWP fines at ambient temperature for 8 minutes with $2000 \mathrm{rpm}$ agitation. The resulting mixture was then subjected to $4000 \mathrm{rpm}$ centrifugation at $10{ }^{\circ} \mathrm{C}$ for 10 minutes, which separated soluble, gel and insoluble parts. ${ }^{21}$ Dissolved and undissolved components were neutralized by $\mathrm{H}_{2} \mathrm{SO}_{4}$ (5\% concentration) and poured separately on a weighed filter paper and washed with distilled water and acetone, respectively. These components collected on filter paper were dried overnight at $70{ }^{\circ} \mathrm{C} .^{3}$

Moreover, to investigate the performance of different dosages of urea in the mentioned system, three levels of weight ratio of urea:sodium hydroxide (i.e. 4:7, 7:7 and 12:7) in the aqueous medium were considered based on previous studies ${ }^{7,22}$ and applied as 
solvents in a constant ratio of 3:100 fiber fines:solvent (dry weight).

Furthermore, the influence of the weight ratio of fines to the solvent $(1: 100,3: 100$ and $5: 100$ at the optimal level of urea:sodium hydroxide ratio) was also considered. Figure 1 summarizes the different steps of the solving process.

Finally, the percent of cellulose dissolution $\left(S_{a}\right)$ was calculated by the following formula (Eq. (1)): ${ }^{3}$

$\mathrm{S}_{\mathrm{a}}=\left[\mathrm{W}_{1} /\left(\mathrm{W}_{1}+\mathrm{W}_{2}\right)\right] \times 100$

where $S_{\mathrm{a}}$ : percentage of cellulose dissolution, $\mathrm{W}_{1}$ : the dry weight of dissolved cellulose, $\mathrm{W}_{2}$ : total weight of undissolved cellulose.

\section{Determining the degree of polymerization of dissolved cellulose (DP)}

The degree of polymerization of cellulose was determined based on the viscosity measurement. The viscosity of the cellulose solution was assessed according to the standard TAPPI T230 om-08 in a viscosity bath at a temperature of $25^{\circ} \mathrm{C}$. In this regard, the viscosity of the $0.5 \%$ cellulose solution was obtained using $0.5 \mathrm{M}$ copper ethylene diamine (CED) as a solvent in the tube of the viscometer. Then, the degree of polymerization was calculated considering the determined viscosity according to SCAN-CM 15:88 standard with the following formula (Eq. (2)): $[\eta]=\mathrm{Q} . \mathrm{DP}^{\mathrm{a}}$

where $\eta$ : viscosity, Q: constant value equal to 2.28 , DP: degree of polymerization, a: constant value equal to 0.76 .
Field emission scanning electron microscopy (FESEM) of the cellulose film

The dissolved cellulose film was observed by the field emission scanning electron microscopy (FESEM) technique, using a Mira3 XMU FE-SEM apparatus (TESCAN, Czech Republic), at an accelerating voltage of $10 \mathrm{kV}$, at various magnifications. For this purpose, the surface of the biofilm was covered with a thin layer of gold $(10 \mathrm{~nm})$ and the film was fixed with silver glue on specific plates.

\section{Determination of cellulose crystallinity with X-ray diffraction $(X R D)$}

To evaluate the crystallinity of dissolved cellulose, a Philips X'Pert MPD diffractometer (Eindhoven, Netherlands), with $\mathrm{Cu} \mathrm{Ka}$ radiation at the wavelength of $0.15405 \mathrm{~nm}$ at $40 \mathrm{kV}$ and $40 \mathrm{~mA}$, was used. The diffracted intensity was analyzed in the range from $2 \theta$ $=8^{\circ}$ to $59^{\circ}$, with the step size of $0.04^{\circ}$ at 0.8 s per step. The crystallinity index ( $\mathrm{CrI})$ of the dissolved cellulose was determined according to Segal's formula ${ }^{23}$ (Eq. (3)):

$$
\text { CrIs }=\frac{I_{0 N z}-I_{\operatorname{mm}}}{I_{00 z}} \times 100
$$

where $\mathrm{I}_{002}$ : the highest intensity in-network diffraction (002), (2 $\theta$ for cellulose type-I at $22.7^{\circ}$ and regenerated cellulose at $\left.21.7^{\circ}\right) ; \mathrm{I}_{\mathrm{am}}$ : intensity in the network diffraction of the amorphous region ( $2 \theta$ for cellulose type-I at $18^{\circ}$ and regenerated cellulose at $16^{\circ}$ ).
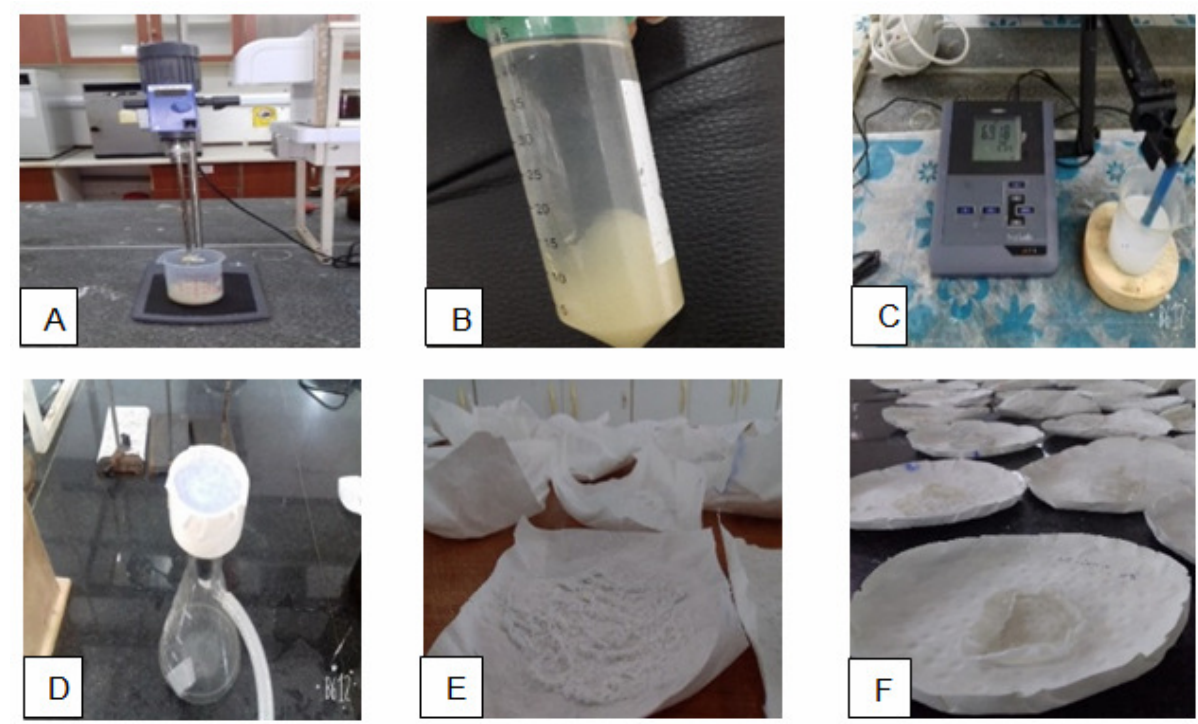

Figure 1: Dissolving process: A: Mixing of fines with an aqueous solvent, B: Dissolved and undissolved products after centrifugation, C: Neutralization with sulfuric acid, D: Washing dissolved and undissolved products, E: Dried undissolved part, F: Dried dissolved part

Fourier-transform infrared analysis of dissolved part

FTIR spectroscopy was performed to gather more data about the dissolved part. For this purpose, the dissolved part and the fiber fines were prepared with $\mathrm{KBr}$ and their IR spectra were compared. A Shimadzu FTIR 8400s (Shimadzu, Kyoto, Japan) was used for 
the FTIR analysis. The range from 4000 to $400 \mathrm{~cm}^{-1}$ was examined at a resolution of $4 \mathrm{~cm}^{-1}$ with 32 scans.

\section{RESULTS AND DISCUSSION Chemical composition of RPWP fines}

The results showed that the recycled fiber fines consist of $1.89 \%$ ash, $0.04 \%$ extractable materials and $98.07 \%$ holocellulose (cellulose and hemicelluloses). As can be observed, the content of extractable materials was very low, which is explained by no notable extractives and contaminants existing in this grade of wastepaper. Also, considering the washing process for collecting fiber fines on the 400 mesh screen, the ash content was insignificant $(1.89 \%)$. As the printing and writing pulp was a bleached one, the lignin content was considered to be very low and therefore the remaining part was attributed to holocellulose. The lack of lignin was confirmed by FTIR analysis which will be presented subsequently.

\section{Dissolving fiber fines with the urea/sodium hydroxide system}

The results displayed in Figure 2 confirmed the successful performance of the sodium hydroxide solution (7\% concentration) in dissolving the RPWP fines. According to previous studies, ${ }^{24}$ sodium hydroxide itself can dissolve cellulose, resulting in dissolved cellulose with low DP, by breaking the parts of cellulose hydrogen bonds with $\mathrm{OH}$ groups of $\mathrm{NaOH}$ and stabilizing the hydrophilic hydroxyl groups with $\mathrm{Na}^{+}$.

The application of urea along with sodium hydroxide (with 7:4 sodium hydroxide:urea ratio) has led to a significant increase in the dissolution of RPWP fines, with an about $33 \%$ gain compared to the application of sodium hydroxide alone.

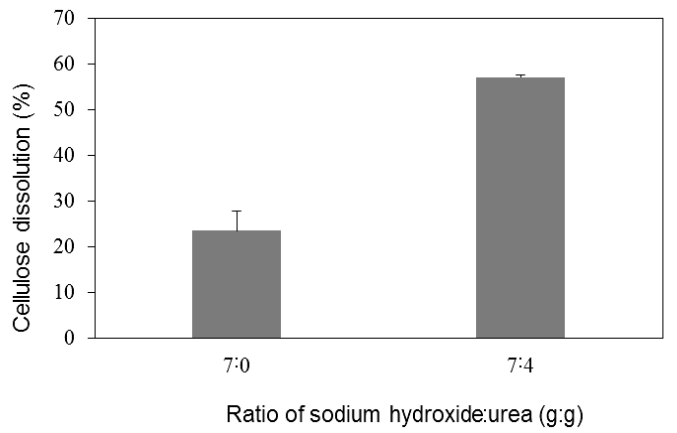

Figure 2: Effect of applying sodium hydroxide alone and the sodium hydroxide:urea system in dissolving RPWP fines
This result was consistent with the other reported findings. ${ }^{22}$ It seems that urea can prevent dissolved cellulose chains from re-association and therefore it contributes to the stabilization of the cellulose solution, which surely affects the dissolving rate.

\section{Effect of urea dosages on the dissolution process}

Figure 3 shows the recycled fines solubility for different levels of urea consumption at a constant ratio of solvent to dry weight of the fines (ratio $3: 100$ ). As can be seen, the increase in the urea dosage in the aqueous solution had no significant effect on the dissolution of fines. It has been previously reported that, with increasing the dosage of urea, part of $\mathrm{NaOH}$ may react with urea instead of cellulose, which would probably reduce the dissolution efficiency. ${ }^{22}$

\section{Effect of fines concentration on dissolution process}

Figure 4(A) shows the effect of the RPWP fines concentration on their dissolution at the fixed level of sodium hydroxide:urea. According to the obtained results, by increasing the proportion of fines in the fines:solvent ratio, the performance of the solvent would reduce significantly. Higher biomass will lead to less access of the chemicals to the cellulosic fines and thus less chemical reaction between the solvent and the fines, which certainly decreases the dissolution.

Moreover, Figure 4(B) shows the effect of recycled fiber size on the performance of sodium hydroxide:urea at a fixed weight ratio of urea:sodium hydroxide $(7: 4(\mathrm{~g}: \mathrm{g}))$ and a fixed amount of recycled fibers/fines $(1 \mathrm{~g})$.

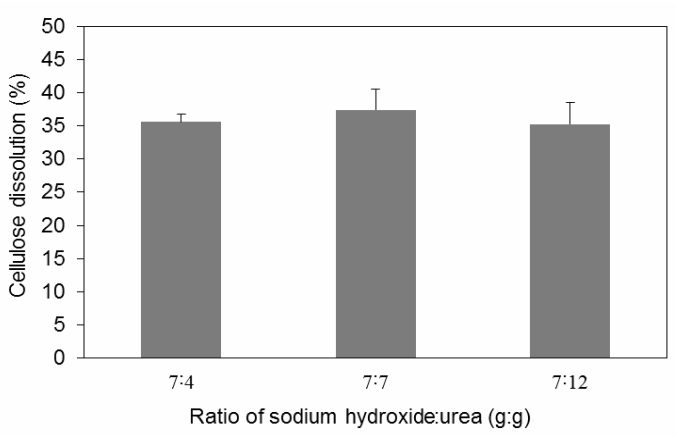

Figure 3: Dissolution of recycled fines in different ratios of the sodium hydroxide:urea system

$(\mathrm{g}: \mathrm{g})$ 

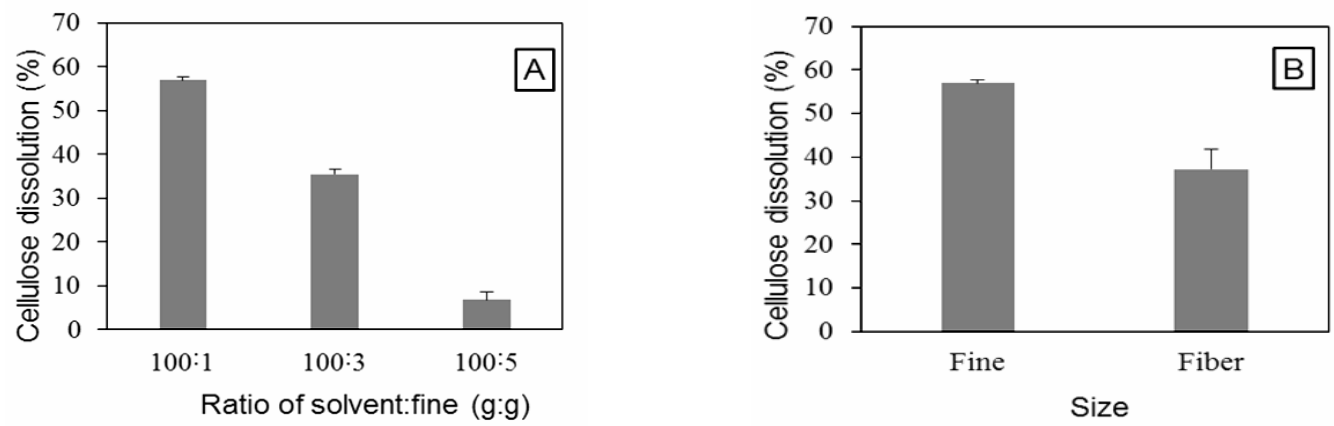

Figure 4: (A) Effect of recycled fines:solvent ratio on cellulose dissolution, (B) effect of fiber size on system performance

As can be seen, the dissolution of fines $(76 \mu$ length) in the aqueous system is higher than that of fibers. It seems that the cellulosic fines have a special surface area, compared to the original fibers, so more hydroxyl groups are available in the access of the aqueous system (especially $\mathrm{OH}^{-}$ and $\mathrm{Na}^{+}$of the sodium hydroxide), which allow alkali to penetrate for a better reaction.

\section{Dissolved cellulose crystallinity}

Figure 5 shows the XRD results for evaluating the crystallinity of RPWP fines, compared to those of dissolved celluloses prepared with different treatments. Accordingly, the specific peaks at $22.5^{\circ}$ and $16.5^{\circ}$, which can be observed in all the treatments, are related to cellulose type-I (natural cellulose). ${ }^{25}$ Also, in RPWP fines, a sharp peak at $29.5^{\circ}$ indicates the presence of minerals (related to mineral fillers in the wastepaper). However, in all the dissolved celluloses prepared with different treatments, this sharp peak at $29.5^{\circ}$ disappeared, which is probably explained by the removal of the fillers by the washing and centrifuging processes during cellulose dissolution.

Also, a comparison of the dissolved cellulose with RPWP fines showed that the peak at $22.5^{\circ}$ was changed into two separate peaks, which have shifted to the left to $20.3^{\circ}$ and $21.59^{\circ}$. Also, the $15.5^{\circ}$ peak has shifted to $12.28^{\circ}$. The mentioned changes can be related to converting cellulose type-I to cellulose type-II through dissolving RPWP fines with the urea/sodium hydroxide systems and cellulose regeneration. ${ }^{7,26}$

Furthermore, the calculated crystallinity for RPWP fines and dissolved samples are shown in Table 1. As can be observed, the crystallinity of the fines was higher than that of the dissolved samples. This reduction in the crystallinity of the dissolved samples can be attributed to the performance of the urea/sodium hydroxide system in the dissolving process. It seems that $\mathrm{NaOH}$ molecules penetrate the crystalline region of the cellulose chains and break the hydrogen bonds in them, which results in cellulose regeneration, reducing its crystallinity. ${ }^{7}$

Also, at constant usage of chemicals in the dissolving process, there was no obvious difference between the crystallinity of dissolved celluloses obtained from 1\% RPWP fines and 3\% RPWP fines concentration, but the 5\% RPWP fines concentration showed an increase in crystallinity. Thus, it can be concluded that, at constant dosage of solvent, the higher the concentration of fines, the less efficient is the dissolving process and the higher is cellulose crystallinity.

Moreover, at a constant level of fines ( $3 \%$ base on dry weight), an increment in the urea dosage increased the crystallinity of dissolved celluloses. Although the presence of urea in the process of alkaline dissolution is necessary, its excessive dosage can reduce the dissolution rate, resulting in higher cellulose crystallinity. Urea can prevent the reconnection of cellulosic chains after dissolution in the alkaline system. ${ }^{15}$ However, excessive amounts of urea may involve part of the sodium hydroxide, and this phenomenon would disturb the dissolving process of cellulose. ${ }^{22}$

\section{FTIR analysis of RPWP fines and dissolved cellulose}

Figure 6 shows the FTIR spectra of RPWP fines, as well as those of the dissolved celluloses with different urea/sodium hydroxide treatments. According the obtained results, several peaks can be observed in the FTIR spectra. 
SHAKIBA BAGHERI et al.

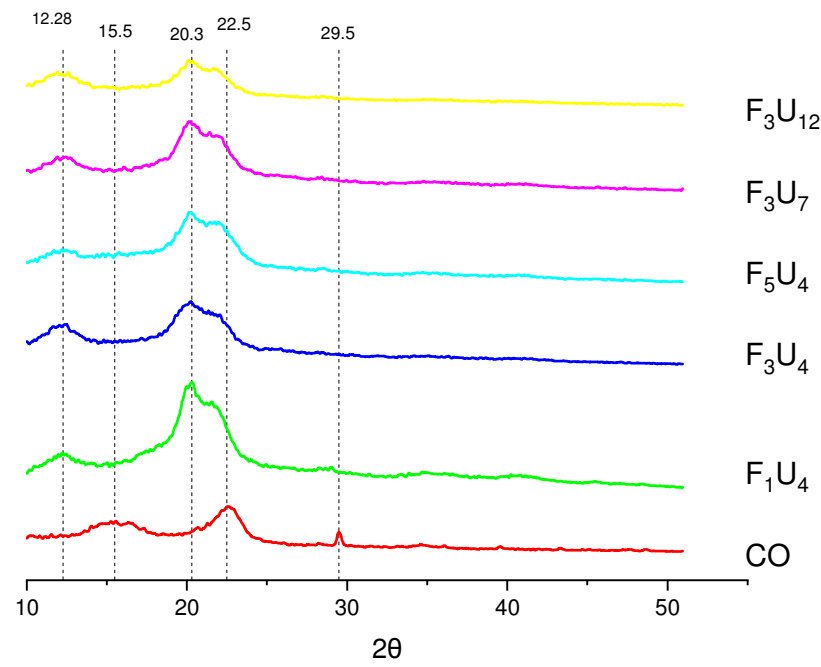

Figure 5: X-ray patterns of RPWP fines and dissolved celluloses prepared with different urea/sodium hydroxide systems $\left(\mathrm{F}_{3} \mathrm{U}_{12}: 3 \%\right.$ RPWP fines $/ 12 \%$ urea, $\mathrm{F}_{3} \mathrm{U}_{7}: 3 \%$ RPWP fines/7\% urea, $\mathrm{F}_{5} \mathrm{U}_{4}: 5 \%$ RPWP fines $/ 4 \%$ urea, $\mathrm{F}_{3} \mathrm{U}_{4}: 3 \%$ RPWP fines/4\% urea, $\mathrm{F}_{1} \mathrm{U}_{4}: 1 \%$ RPWP fines $/ 4 \%$ urea, $\mathrm{CO}$ : RPWP fines)

Table 1

Crystallinity of RPWP fines and dissolved samples in the urea/sodium hydroxide aqueous system

\begin{tabular}{lc}
\hline Treatment & Crystallinity (\%) \\
\hline RPWP fines & 71.5 \\
1\% Fines dissolved in 4\% urea/7\% sodium hydroxide & 50.42 \\
3\% Fines dissolved in 4\% urea/7\% sodium hydroxide & 49.77 \\
5\% Fines dissolved in 4\% urea/7\% sodium hydroxide & 54.57 \\
3\% Fines dissolved in 7\% urea/7\% sodium hydroxide & 51.31 \\
3\% Fines dissolved in 12\% urea/7\% sodium hydroxide & 56.55 \\
\hline
\end{tabular}

Considering all the treatments, the presence of a broad peak in the range of $3100-3600 \mathrm{~cm}^{-1}$ is common for lignocellulosic materials related to the stretch vibration of hydroxyl groups ${ }^{27-29}$ and the existence of intramolecular hydrogen bonds of type $\mathrm{O}_{(2)} \mathrm{H} \ldots \mathrm{O}_{(6)}$ in cellulose. ${ }^{30}$ It should be mentioned that this peak in celluloses dissolved in the urea/ $\mathrm{NaOH}$ aqueous systems has a slight shift to the left (about $3429 \mathrm{~cm}^{-1}$ ), which can be related to converting cellulose type-I to cellulose type-II through dissolving RPWP fines with urea/sodium hydroxide systems and cellulose regeneration. ${ }^{31}$

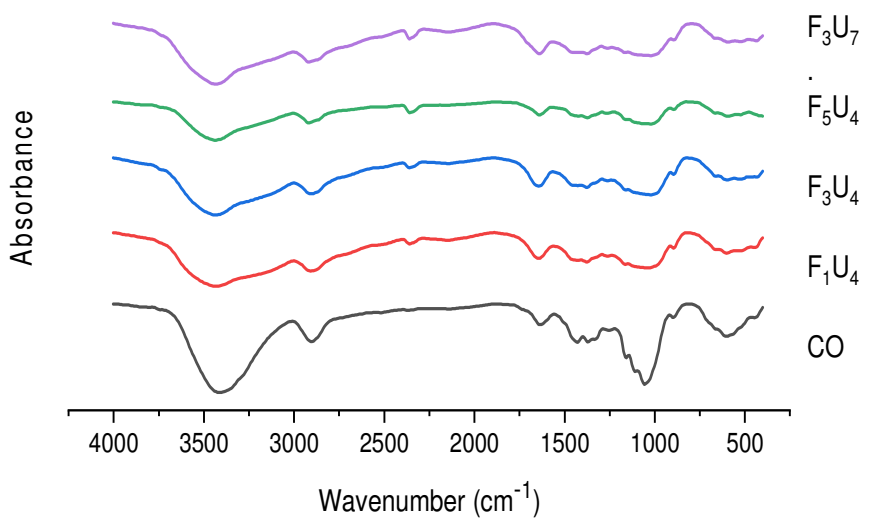

Figure 6: FTIR spectra of RPWP fines and dissolved celluloses obtained with different urea/sodium hydroxide systems $\left(\mathrm{F}_{3} \mathrm{U}_{7}: 3 \%\right.$ RPWP fines/7\% urea, $\mathrm{F}_{5} \mathrm{U}_{4}: 5 \%$ RPWP fines $/ 4 \%$ urea, $\mathrm{F}_{3} \mathrm{U}_{4}: 3 \%$ RPWP fines/4\% urea,

$\mathrm{F}_{1} \mathrm{U}_{4}: 1 \%$ RPWP fines/4\% urea, $\mathrm{CO}$ : RPWP fines) 
In all the treatments, the peak at 2897-2920 $\mathrm{cm}^{-1}$ can be attributed to the stretch vibration of $\mathrm{C}-\mathrm{H}$ in cellulose molecule. ${ }^{28-29,32}$ Moreover, the presence of a peak at $2359 \mathrm{~cm}^{-1}$ is probably an error related to the existence of $\mathrm{CO}_{2}$ in the prepared samples for FTIR assessment. The absence of a peak at about $1700 \mathrm{~cm}^{-1}$ in all the treatments (even fines as a control sample) indicates the absence of carboxylic groups related to hemicelluloses. ${ }^{29}$ This is acceptable due to the probable removal of hemicelluloses from RPWP fines and dissolved cellulose during various processes, such as pulping, washing and dissolving. The observed peak at $1614-1645 \mathrm{~cm}^{-1}$ wavelengths in all the treatments may be related to the presence of moisture in the samples prepared for spectroscopy. ${ }^{31}$ Furthermore, the peak at $1429 \mathrm{~cm}^{-1}$, which is clearly defined in the control sample compared with the treated samples, can be referred to as the bending of the $\mathrm{CH}_{2}$ related to $\mathrm{C}-6$ in the cellulose molecule. ${ }^{27}$ The peak appearing at $1370 \mathrm{~cm}^{-1}$ can be assigned to $\mathrm{C}-\mathrm{H}$ bending vibration in cellulose. Also, the peak at the wavelength of $1050 \mathrm{~cm}^{-1}$ can be related to $\mathrm{CO}$-stretching in the $\mathrm{C}-\mathrm{O}-(\mathrm{H})$ bond, especially $\mathrm{C}_{(3)} \ldots \mathrm{O}_{(3)} \mathrm{H}$ in cellulose. ${ }^{29,31}$ Moreover, the observed peak at $893-897 \mathrm{~cm}^{-1}$ may be assigned to $\mathrm{C}-\mathrm{O}-\mathrm{C}$ ( $\beta$-glycoside bond) in the cellulose. ${ }^{31,33}$ A peak in the range of $700-500 \mathrm{~cm}^{-1}$ can be referred to as the bending vibration of the $\mathrm{O}-\mathrm{H}$ bond (out of plane) in the cellulose molecule. ${ }^{31}$ As can be seen, the intensity of this peak is high in the RPWP fines and, contrariwise, very low in the dissolved samples.

Considering the FTIR results for the RPWP fines and dissolved celluloses with different treatments, it can be observed that the intensity of the peaks at 3439-3414, 2920-2897, 1050, and $700-500 \mathrm{~cm}^{-1}$ have reduced significantly in the spectra of dissolved celluloses in comparison with the control sample (fiber fines). It seems that converting cellulose type-I to cellulose type-II is the reason for the mentioned results. In other words, cellulose type-I would experience molecular re-arrangement by penetrating the sodium hydroxide. ${ }^{27}$ Moreover, the results of infrared spectroscopy confirmed the existence of no lignin in the structure of RPWP fines and in the dissolved celluloses.

\section{FE-SEM images of cast dissolved cellulose}

Figure 7 shows the image of the dissolved cellulose prepared by applying 7\% sodium hydroxide/4\% urea/89\% water on 1 g RPWP fines. The uniform structure of the cast dissolved cellulose film confirms the proper solubility of the RPWP fines in the mentioned system.

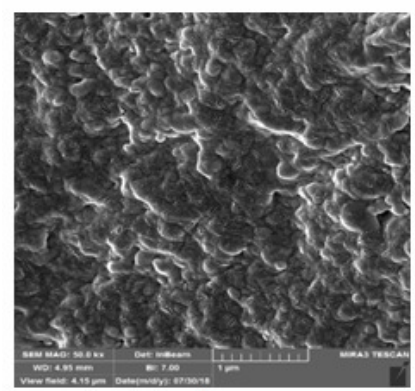

A

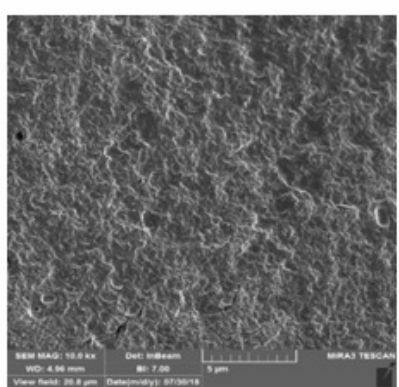

B

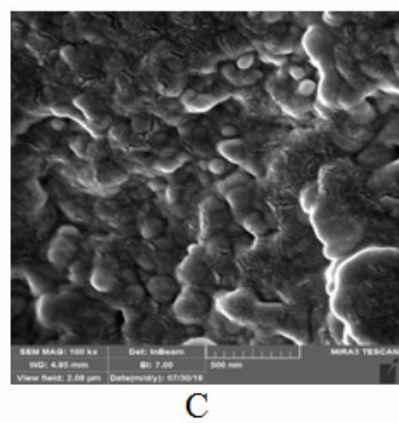

C

Figure 7: FE-SEM images of cast dissolved cellulose with different magnitudes

(A: $10 \mathrm{kx}, \mathrm{B}: 50 \mathrm{kx}$ and C: $100 \mathrm{kx}$ )

\section{DP of dissolved cellulose}

One of the main factors affecting cellulose dissolution is its degree of polymerization. ${ }^{34}$ The DP of cellulose is defined as the number of glucose units in the cellulose chain. ${ }^{35}$ The DP of RPWP cellulose and the dissolved cellulose $(1 \mathrm{~g}$ RPWP fines dissolved by applying $7 \%$ sodium hydroxide $/ 4 \%$ urea/ $89 \%$ water) was about 637 and 521, respectively. As can be observed, through the dissolving process, the cellulose DP was not significantly reduced. In this respect, another report claimed similar results. ${ }^{6}$ This means that the applied dissolving process in the current research (urea/sodium hydroxide system), in contrast to some other methods, does not break the cellulose chains. This achievement is important in applications that need cellulose with a higher DP. 
Furthermore, in contrast with the $\mathrm{NaOH}$ aqueous solution, which dissolves lignocellulosic biomaterials by reducing the cellulose DP to less than $400,{ }^{24}$ it seems that applying urea/ $\mathrm{NaOH}$ can successfully dissolve cellulose with a lower decrease in the DP. ${ }^{36}$ Also, the cellulose solution obtained by applying $\mathrm{NaOH}$ was not so stable and easily changed to a gel form. This phenomenon is probably explained by the high tendency of the hydrophobic parts of dissolved cellulose for rejoining. ${ }^{22}$ As mentioned before, the addition of urea improves cellulose dissolution, even with higher DP, by improving the performance of sodium hydroxide. It should be kept in mind, however, that excessive amounts of urea can lead to a sharp decline in the dissolving process. ${ }^{37}$

\section{CONCLUSION}

Fiber fines (as a low value waste of papermaking mills) can be considered as a beneficial lignocellulosic material for producing value-added bio-products. This research tried to consider the urea/sodium hydroxide aqueous system as an environmentally friendly and inexpensive method for dissolving recycled fiber fines. The results indicated a very significant effect of urea on the performance of sodium hydroxide in dissolving the recycled fiber fines. However, it should be taken into account that an excessive amount of urea and its unbalanced ratio with sodium hydroxide may negatively affect the dissolution of fines. Moreover, reducing the concentration of fines in the suspension increased their solubility. The FTIR spectra confirmed the presence of cellulose, without any hemicelluloses and lignin, in the dissolving part. The X-ray diffraction analysis of RPWP fines before and after dissolution indicated cellulose regeneration from native cellulose to cellulose type-II. Also, the crystallinity for RPWP fines was higher than that of the dissolved cellulose, which could be attributed to cellulose regeneration. Furthermore, the dissolving process did not have any specific effect on DP reduction, which can be an important advantage over other conventional dissolving methods. Also, the well-integrated cellulosic biofilm structure confirmed by FE-SEM images can be attributed to the successful dissolution of RPWP fines in the urea/sodium hydroxide aqueous system.

ACKNOWLEDGEMENTS: The authors would like to thank the Deputy Dean for Research of the Natural Resources Faculty of Tarbiat Modares
University (TMU) for supporting the current research. Also, the authors are grateful to Dr. M. Taghi Asadollahzadeh and Eng. S. Behzad Hosseini, the lab directors of the Wood and Paper Science Department for their kind help during laboratory works.

\section{REFERENCES}

1 J. Zhou and L. Zhang, Polym. J., 32, 866 (2000), https://doi.org/10.1295/polymj.32.866

2 Z. Shi, Q. Yang, J. Cai, S. Kuga and Y. Matsumoto, Cellulose, 21, 1205 (2014), https://doi.org/10.1007/s10570-014-0226-2

3 L. Zhang, D. Ruan and S. Gao, J. Polym. Sci. B Polym. Phys., 40, $1521 \quad$ (2002), https://doi.org/10.1002/polb.10215

4 D. Klemm, B. Heublein, H. P. Fink and A. Bohn, Angew. Chem. Int. Ed., 44, 3358 (2005), https://doi.org/10.1002/anie.200460587

5 B. Medronho and B. Lindman, Curr. Opin. Colloid Int., $\quad \mathbf{1 9}, \quad 3240 \quad$ (2014), https://doi.org/10.1016/j.cocis.2013.12.001

6 J. Cai, L. Zhang, J. Zhou, H. Qi, H. Chen et al., Adv. Mater., 19, $821 \quad$ (2007), https://doi.org/10.1002/adma.200601521

7 R. Li, S. Wang, A. Lu and L. Zhang, Cellulose, 22, 339 (2015), https://doi.org/10.1007/s10570-014-05426

8 E. Amiri, M. Rahmaninia and A. Khosravani, BioResources, 14, 7687 (2019)

9 M. C. Monte, E. Fuente, A. Blanco and C. Negro, Waste Manage., 29, $293 \quad$ (2008), https://doi.org/10.1016/j.wasman.2008.02.002

10 R. Sabazoodkhiz, M. Rahmaninia and O. Ramezani, Cellulose, 24, $3433 \quad$ (2017), https://doi.org/10.1007/s10570-017-1345-3

11 H. Kermanian, Z. Razmpour, O. Ramezani, S. Mahdavi, M. Rahmaninia et al., BioResources, 8, 5424 (2013)

12 M. Rahmaninia and A. Khosravani, Cellulose Chem. Technol., 49, $203 \quad$ (2015), https://www.cellulosechemtechnol.ro/pdf/CCT2(2015) /p.203-208.pdf

${ }^{13}$ B. C. Min, B. V. Bhayani, V. S. Jampana and B. V. Ramarao, Bioresour. Bioprocess, 2, 1 (2015), https://doi.org/10.1186/s40643-015-0068-2

14 Q. Yang, H. Qi, A. Lue, K. Hu, G. Cheng et al., Carbohyd. Polym., 83, $1185 \quad$ (2011), https://doi.org/10.1016/j.carbpol.2010.09.020

15 Z. Jiang, Y. Fang, J. Xiang, Y. Ma, A. Lu et al., J. Phys. Chem. B, 118, $10250 \quad$ (2014), https://doi.org/10.1021/jp501408e

16 H. P. Fink, P. Weigel, H. J. Purz and J. Ganster, Prog. Polym. Sci., 26, 1341 (2001), https://doi.org/10.1016/S0079-6700(01)00025-9

17 A. Isogai and R. H. Atalla, Cellulose, 5, 309 (1998), https://doi.org/10.1023/A:1009272632367

18 C. Yamane, M. Mori, M. Saito and K. Okajima, 
Polym. J., $\quad$ 28, 1039 https://doi.org/10.1295/polymj.28.1039

19 M. Ghasemi, P. Alexandridis and M. Tsianou, Biomacromolecules, $\quad$ 19, $640 \quad$ (2018), https://doi.org/10.1021/acs.biomac.7b01745

20 S. M. H. Sangtarashani, M. Rahmaninia, R. Behrooz and A. Khosravani, J. Environ. Manage., 270, 110853 https://doi.org/10.1016/j.jenvman.2020.110853

21 J. Cai, L. Zhang, J. Zhou, H. Li, H. Chen et al., Macromol. Rapid Commun., 25, 1558 (2004), https://doi.org/10.1002/marc.200400172

22 B. Xiong, P. Zhao, K. Hu, L. Zhang and G. Cheng, Cellulose, 21, 1183 (2014), https://doi.org/10.1007/s10570-014-0221-7

23 L. Segal, J. J. Creely, Jr. A. E. Martin and C. M. Conrad, Text. Res. J., 29, 786 (1959), https://doi.org/10.1177/004051755902901003

24 B. Łaskiewicz, J. Appl. Polym. Sci., 67, 1871 (1998), https://doi.org/10.1002/(SICI)10974628(19980314)67:11<1871::AID-APP5>3.0.CO;2-I

25 F. Niroomand, A. Khosravani and H. Younesi, Cellulose, 23, $1311 \quad$ (2016), https://doi.org/10.1007/s10570-016-0872-7

26 H. Qi, C. Chang and L. Zhang, Cellulose, 15, 779 (2008), https://doi.org/10.1007/s10570-008-9230-8

27 M. L. Nelson and R. O'Connor, J. Appl. Polym. Sci., $\quad \mathbf{8}, \quad 1325 \quad$ (1964), https://doi.org/10.1002/app.1964.070080323
28 K. K. Pandey, J. Appl. Polym. Sci., 1, 1969 (1999), https://doi.org/10.1002/(SICI)1097-

4628(19990321)71:12<1969::AID-APP6>3.0.CO;2-D

29 H. Yang, R. Yan, H. Chen, D. H. Lee and C. Zheng, Fuel, 86, $1781 \quad$ (2007), https://doi.org/10.1016/j.fuel.2006.12.013

30 A. M. Olsson and L. Salmén, Carbohyd. Res., 339, 813 https://doi.org/10.1016/j.carres.2004.01.005

31 J. Siroky, R. S. Blackburn, T. Bechtold, J. Taylor and P. White, Cellulose, 17, 103 (2010), https://doi.org/10.1007/s10570-009-9378-X

32 L. Zhang, J. Zhou and L. Zhang, Carbohyd. Polym., $\quad 94, \quad 386 \quad$ (2013), https://doi.org/10.1016/j.carbpol.2012.12.077

33 S. Y. Oh, D. I. Yoo, Y. Shin, H. C. Kim, H. Y. Kim et al., Carbohyd. Res., 40, 2376 (2005), https://doi.org/10.1016/j.carres.2005.08.007

34 D. L. Morgado, E. Frollini, A. Castellan, D. S. Rosa and V. Coma, Cellulose, 18, 699 (2011), https://doi.org/10.1007/s10570-011-9516-0

35 C. Campano, A. Balea, A. Blanco and C. Negro, Cellulose, 23, 57 (2016), https://doi.org/10.1007/s10570-015-0802-0

36 B. J. C. Duchemin, PhD Dissertation, University of Canterbury, Canterbury, New Zealand, 2008, http://dx.doi.org/10.26021/1603

37 R. Gavillon, PhD Dissertation, Paris Institute of Technology, Paris, France, 2007 